\title{
Collapse of Randomly Coiled Schizophyllan in Mixtures of Water and Dimethylsulfoxide ${ }^{\dagger}$
}

\author{
Takahiro SATO, Kazuo SAKURAI, Takashi NorisUYe, \\ and Hiroshi FuJITA \\ Department of Macromolecular Science, Osaka University, \\ Toyonaka, Osaka 560, Japan
}

(Received October 27, 1982)

\begin{abstract}
Dimethylsulfoxide (DMSO) solutions of schizophyllan were diluted with water to a series of $w_{\mathrm{H}}$ (the water weight fraction in the mixed solvent), and the subsequent changes in viscosity were measured as a function of $w_{\mathrm{H}}$, polymer concentration $c$, and time $t$. As $w_{\mathrm{H}}$ was increased, $\left(\ln \eta_{\mathrm{r}}\right) / c$ at fixed $c$ and $t(10 \mathrm{~h})$ remained constant up to $w_{\mathrm{H}} \sim 0.13$, then underwent a sharp decrease, passed through a deep minimum at $w_{\mathrm{H}} \sim 0.25$, and increased markedly. The intrinsic viscosities of five schizophyllan samples (ranging in molecular weight from $1.35 \times 10^{4}$ to $1.5 \times 10^{5}$ ) determined at $w_{\mathrm{H}}=0.25$ were essentially independent of molecular weight, being about $8 \mathrm{~cm}^{3} \mathrm{~g}^{-1}$. An attempt was made to explain the entire experimental finding as a result of combined effects of two molecular processes: collapse of random coils to globules by intrachain hydrogen bonding and formation of aggregates by interchain hydrogen bonding.
\end{abstract}

KEY WORDS Polysaccharide / Schizophyllan / Globule / Chain Collapse /

Intrinsic Viscosity / Intermolecular Association / Intramolecular Association /

Schizophyllan is an extracellular $\beta$-1,3-D-glucan produced by a fungus schizophyllum commune. As shown in our previous papers, ${ }^{1-3}$ this polysaccharide dissolves in water as a rodlike triple helix, while it disperses in dimethylsulfoxide (DMSO) as a single random coil swollen by excluded-volume effect. Furthermore, schizophyllan directly dissolved in water+DMSO mixures undergoes an all-or-none dissociation from the triple helix to single chains when the weight fraction $w_{\mathrm{H}}$ of water in the binary mixture is lowered to about 0.13 at $25^{\circ} \mathrm{C}^{4}$ or when the solution of $w_{\mathrm{H}}$ $\sim 0.13$ is heated. ${ }^{5}$

In Part $\mathbf{I},{ }^{1}$ we examined by viscometry whether the triple helix would be reformed from single schizophyllan chains dispersed in DMSO when water was added to the solvent to $w_{\mathbf{H}}=0.90$. The data showed no evidence other than the formation of random aggregates. Recently, we attempted a further study of this problem, performing viscosity measurements on DMSO solutions diluted with water to various $w_{\mathrm{H}}$ ranging from 0 to 0.6 . It was found that these "water-diluted" solutions exhibited anomalous viscosity behavior which is hardly anticipated from our previous data. ${ }^{1}$ This finding and its interpretation are described in the present paper.

\section{EXPERIMENTAL}

\section{Samples and Preparation of Solutions}

Most of the present data were obtained with the same schizophyllan sample as used in Part V, ${ }^{4}$ which is designated here as $\mathrm{S}-197$, but the data at $w_{\mathrm{H}}=0.20$ and 0.25 were also obtained with four other samples, H-4, E-3, S-164-2, and U-16. Table I summarizes the molecular weights of these five samples and their intrinsic viscosities $[\eta]$ in pure DMSO at $25^{\circ} \mathrm{C}$. The molecular weights of samples S-197 and U-16 were determined by light scattering (see ref 4) and sedimentation equilibrium, respectively, where-

${ }^{\dagger}$ Triple Helix of Schizophyllum commune Polysaccharide in Dilute Solution. Part VI. 
Table I. Molecular weights and intrinsic viscosities of schizophyllan samples in DMSO at $25^{\circ} \mathrm{C}$

\begin{tabular}{lcc}
\hline Sample & {$[\eta] \times 10^{-2}$} & $\begin{array}{c}\text { Molecular } \\
\text { weight } \times 10^{-4}\end{array}$ \\
\cline { 2 - 3 } $\mathrm{cm}^{3} \mathrm{~g}^{-1}$ & 15.0 \\
S-197 & 0.800 & 11.0 \\
H-4 & 0.675 & 7.9 \\
E-3 & 0.535 & 4.5 \\
S-164-2 & 0.366 & 1.35 \\
U-16 & 0.169 & \\
\hline
\end{tabular}

as those of the other samples were estimated from $[\eta]$, using the previously established relation ${ }^{3}$ between $[\eta]$ and weight-average molecular weight for schizophyllan in DMSO.

Sedimentation equilibrium measurements were made on water-diluted solutions of $w_{\mathbf{H}}=0.25$. In evaluating the apparent molecular weight $M_{\text {app}}$, no correction for preferential adsorption was applied to the data, since we found no difference more than $2 \%$ between the partial specific volume measured at a fixed solvent composition $\left(0.621 \mathrm{~cm}^{3} \mathrm{~g}^{-1}\right)$ and that calculated at fixed chemical potentials of the solvent components using our previous data ${ }^{4}$ for the preferential adsorption coefficient.

Water-diluted solutions of schizophyllan with desired $w_{\mathrm{H}}$ and polymer mass concentrations $c$ were prepared by diluting a pure DMSO solution of a known polymer concentration with a calculated amount of the water + DMSO mixture having an appropriate composition. The mixed solvent having the same composition as that in the final solution was prepared separately. In no case were the final solutions diluted with this mixed solvent in order to obtain different polymer concentrations.

\section{Viscometry}

Viscosities of water-diluted solutions in the range of $c$ from $0.92 \times 10^{-2}$ to $0.05 \times 10^{-2} \mathrm{~g} \mathrm{~cm}^{-3}$ were measured at $25^{\circ} \mathrm{C}$ by use of capillary viscometers of the Ubbelohde type relevant for $w_{\mathrm{H}}$ and $c$ of the test solutions. For example, the viscometer chosen for $w_{\mathrm{H}}=0.25$ and $c=0.05 \times 10^{-2} \mathrm{~g} \mathrm{~cm}^{-3}(1.008 \gtrsim$ relative viscosity $\left.\eta_{\mathrm{r}} \gtrsim 1.005\right)$ had the flow times of $1790.2 \pm 0.8 \mathrm{~s}$ for six independently prepared mixed solvents of $w_{\mathrm{H}}=0.25$, and the average value $1790.2 \mathrm{~s}$ was used to calculate $\eta_{\mathrm{r}}$, so that the measured values of $\left(\ln \eta_{\mathrm{r}}\right) / c$ for solutions of $\eta_{\mathrm{r}} \sim 1.005$ were in error of the order of $\pm 10 \%$. The thermostat was maintained at $25 \pm 0.01^{\circ} \mathrm{C}$.

\section{RESULTS}

Figure 1 shows the changes in $\left(\ln \eta_{\mathrm{r}}\right) / c$ with the time $t$ elapsed after DMSO solutions of sample S197 with different $c$ were diluted with water to $w_{\mathrm{H}}=$ 0.168 . All these curves start at the values far below the horizontal segments representing $\left(\ln \eta_{\mathrm{r}}\right) / c$ for the DMSO solutions with $c=0.917 \times 10^{-2}$ and $0.152 \times 10^{-2} \mathrm{~g} \mathrm{~cm}^{-3}$. This implies that as soon as the dilution is made, there occurs a pronounced decrease in viscosity which is too fast to be measured by our experimental method. For the two higher $c$ the initial decrease in $\left(\ln \eta_{\mathrm{r}}\right) / c$ is followed by a monotonic increase, while for the two lower $c$ there is a further decrease in $\left(\ln \eta_{\mathrm{r}}\right) / c$ before a monotonic increase takes place. Thus, in the latter, $\left(\ln \eta_{\mathrm{r}}\right) / c$ passes through a minimum at a certain elapsed time.

Figure 2 depicts the time dependence of $\left(\ln \eta_{\mathrm{r}}\right) / c$ for samples S-197 in water-diluted solutions of different $w_{\mathrm{H}}$ at a fixed $c\left(=0.68 \times 10^{-2} \mathrm{~g} \mathrm{~cm}^{-3}\right)$. Here, each of the filled circles indicates the value of $\left(\ln \eta_{\mathrm{r}}\right) / c$ prior to the dilution with water. The data points for $w_{\mathrm{H}}=0.121$ show no viscosity change with the elapsed time $t$. For $w_{\mathrm{H}}$ between 0.14 and 0.27 there is a discontinuous decrease in $\left(\ln \eta_{\mathrm{r}}\right) / c$ at the beginning, as observed in Figure 1, but the mode of subsequent changes in $\left(\ln \eta_{\mathrm{r}}\right) / c$ varies with $w_{\mathrm{H}}$. Thus, for $w_{\mathrm{H}}=0.140$ and $0.157,\left(\ln \eta_{\mathrm{r}}\right) / c$ decreases further and appears to level off at a constant value, while for $w_{\mathrm{H}}=0.240$ and $0.270,\left(\ln \eta_{\mathrm{r}}\right) / c$ increases monotonically. Finally, for $w_{\mathrm{H}}=0.300$ there occurs a monotonic increase in $\left(\ln \eta_{\mathrm{r}}\right) / c$ following a discontinuous initial rise. This last feature resembles what we observed for water-diluted solutions of $w_{\mathrm{H}}=0.90$ in Part I. ${ }^{1}$

Values of $\left(\ln \eta_{\mathrm{r}}\right) / c$ at given values of $t$ for sample S-197 were estimated as a function of $w_{\mathrm{H}}$ and $c$ by interpolating the measured $\left(\ln \eta_{\mathrm{r}}\right) / c$ vs. $t$ curves. The results at $10 \mathrm{~h}$ are illustrated in Figure 3, along with the $[\eta] v s . w_{\mathrm{H}}$ relation ${ }^{4}$ for DMSO-diluted solutions of the same sample at $25^{\circ} \mathrm{C}$. Here, by a "DMSOdiluted solution" is meant a solution prepared by dissolving a given schizophyllan sample directly in a water + DMSO mixture. This figure displays the most important finding of the present study. With increasing $w_{\mathrm{H}},\left(\ln \eta_{\mathrm{r}}\right) / c$ of water-diluted solutions 


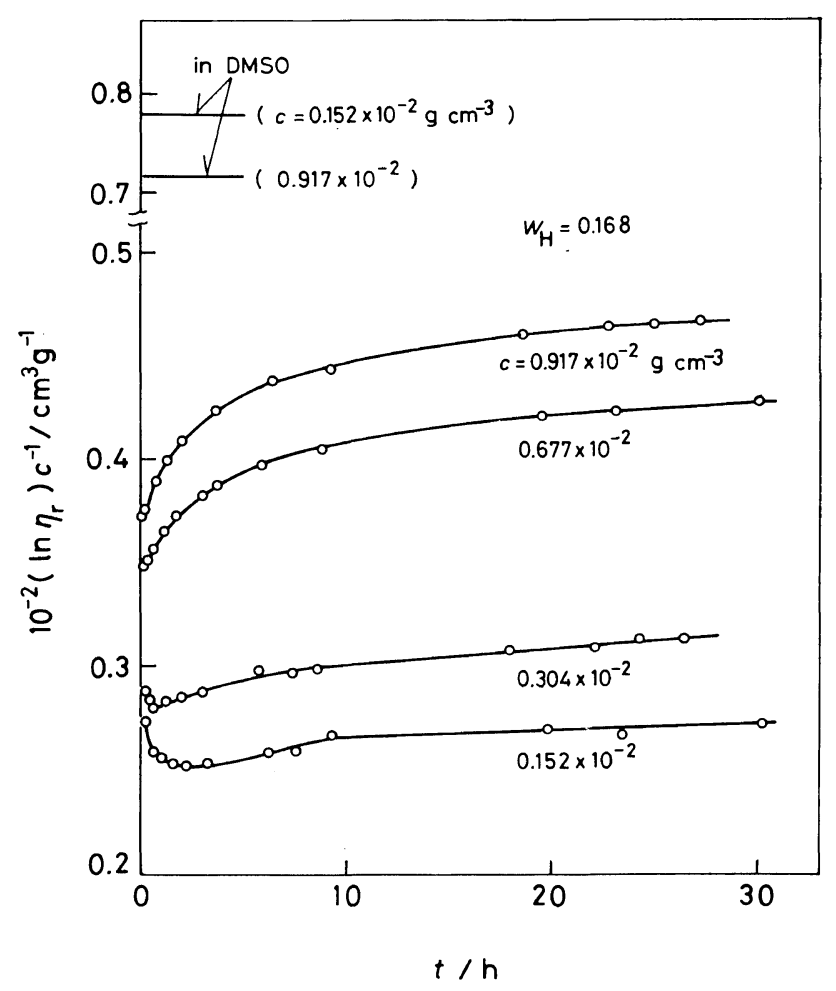

Figure 1. Time dependence of $\left(\ln \eta_{\mathrm{r}}\right) / c$ for a schizophyllan sample S-197 in water-diluted solutions of $w_{\mathrm{H}}=0.168$ at different polymer concentrations. The horizontal segments indicate $\left(\ln \eta_{\mathrm{r}}\right) / c$ of the DMSO solutions with $c=0.917 \times 10^{-2}$ and $0.152 \times 10^{-2} \mathrm{~g} \mathrm{~cm}^{-3}$.

remains constant up to $w_{\mathrm{H}} \sim 0.13$, then abruptly decreases instead of increasing sharply along the $[\eta]$ vs. $w_{\mathrm{H}}$ curve for DMSO-diluted solutions, passes through a deep minimum, and continues to increase markedly. The $\left(\ln \eta_{\mathrm{r}}\right) / c v s . w_{\mathrm{H}}$ relations for waterdiluted solutions at other $t$ showed essentially the same behavior as this. Thus, in the present work, no evidence was obtained for the reformation of the triple helix from molecularly disperse schizophyllan chains in water-diluted solutions. Finally, it is interesting that the sharp decrease in $\left(\ln \eta_{\mathrm{r}}\right) / c$ of water-diluted solutions and that in $[\eta]$ of DMSOdiluted solutions occur at almost the same $w_{\mathrm{H}}$.

\section{DISCUSSION}

\section{General Consideration}

The present viscosity measurements, as those in Part $\mathrm{I},{ }^{1}$ revealed no evidence for the reformation of triple helices from randomly coiled schizophyllan chains when DMSO solutions of this polysac- charide were diluted with water. Instead, it was found that the viscosity of water-diluted solutions changed in an unexpected way as $w_{\mathrm{H}}$ of the mixed solvent was increased. To explain our observed results we begin with considering what happens to schizophyllan single chains in water-diluted solutions.

The triple helix of schizophyllan is stabilized by interchain hydrogen bonds. ${ }^{1}$ It seems reasonable to consider that when this polysaccharide is dispersed molecularly in a DMSO + water mixture, its hydroxyl groups tend to associate with one another, provided the solvent condition is favorable for hydrogen bond formation. In dilute solutions with which we are concerned here, there are few chances for three schizophyllan chains to meet at the same time. Even if it happens and the solvent is very favorable for hydrogen bonding, it should be extremely unlikely that these chains arrange themselves to be hydrogen bonded in a triple helical form. In such a solvent, it is far more likely that the 


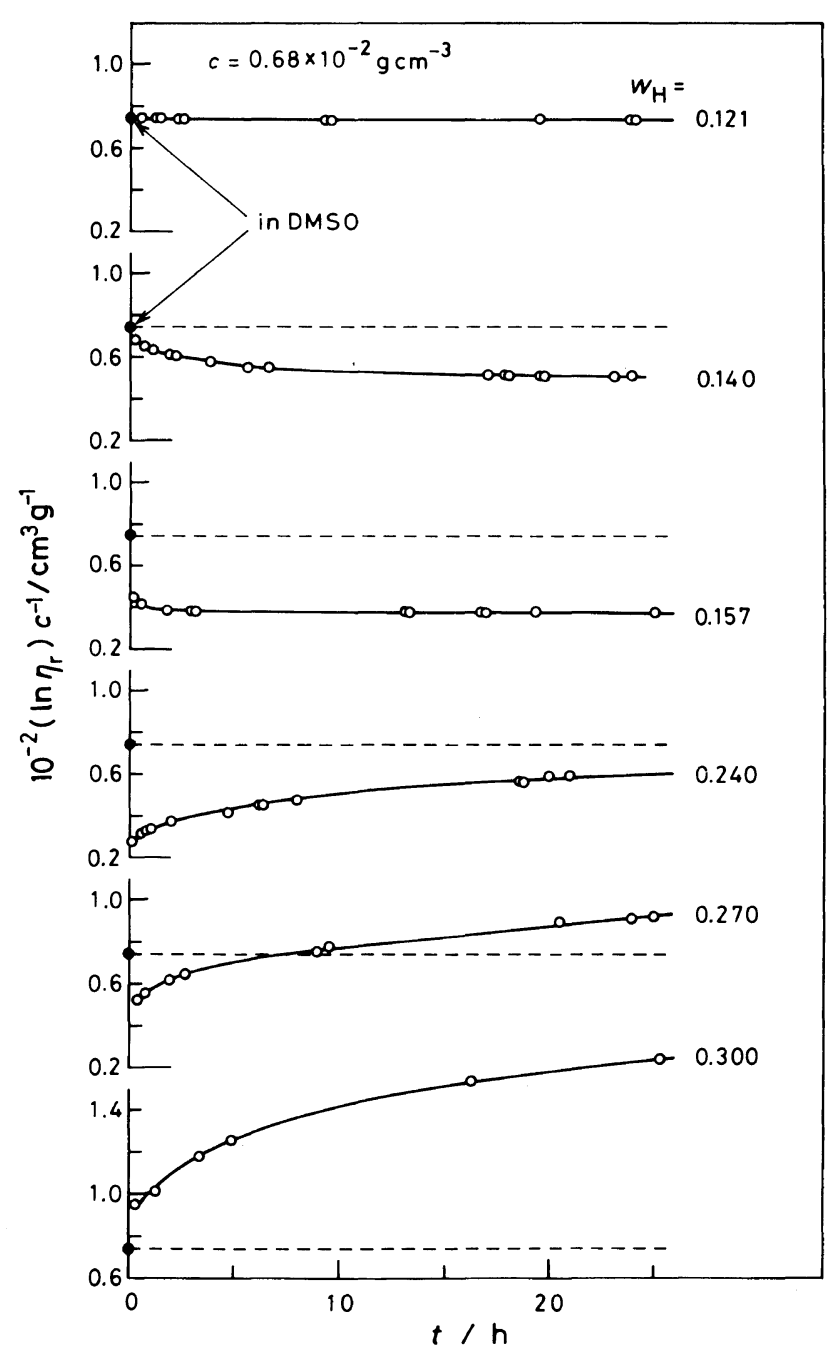

Figure 2. Time dependence of $\left(\ln \eta_{\mathrm{r}}\right) / c$ for sample S-197 in water-diluted solutions of different $w_{\mathrm{H}}$ at a fixed $c$ of $0.68 \times 10^{-2} \mathrm{~g} \mathrm{~cm}^{-3}$. Each of the filled circles represents $\left(\ln \eta_{\mathrm{r}}\right) / c$ of the DMSO solution with the same $c$.

following two processes occur. In one, some of the hydroxyl groups of each randomly coiled chain are hydrogen bonded intramolecularly. This causes the random coil to shrink to a globule of higher segment density, i.e., the so-called chain collapse takes place. In the other, some of the hydroxyl groups of two or more chains, either collapsed or not, are hydrogen bonded intermolecularly, and dimers, trimers, and higher aggregates are formed.

The chain collapse, i.e., intrachain association, decreases the viscosity of the solution, while the formation of aggregates, i.e., interchain association, gives rise to the reverse effect. Either of these associations should be enhanced as the composition of the DMSO + water mixed solvent is changed to a value more favorable for hydrogen bonding, i.e., $w_{\mathrm{H}}$ is increased. The interchain association should be also enhanced with increasing polymer concentration $c$. However, it seems reasonable to assume that, in a first approximation, the collapse of individual random coils takes place independently, i.e., it is independent of $c$. On the basis of these considerations our data delineated in Figures 1 through 3 may be interpreted as follows. 


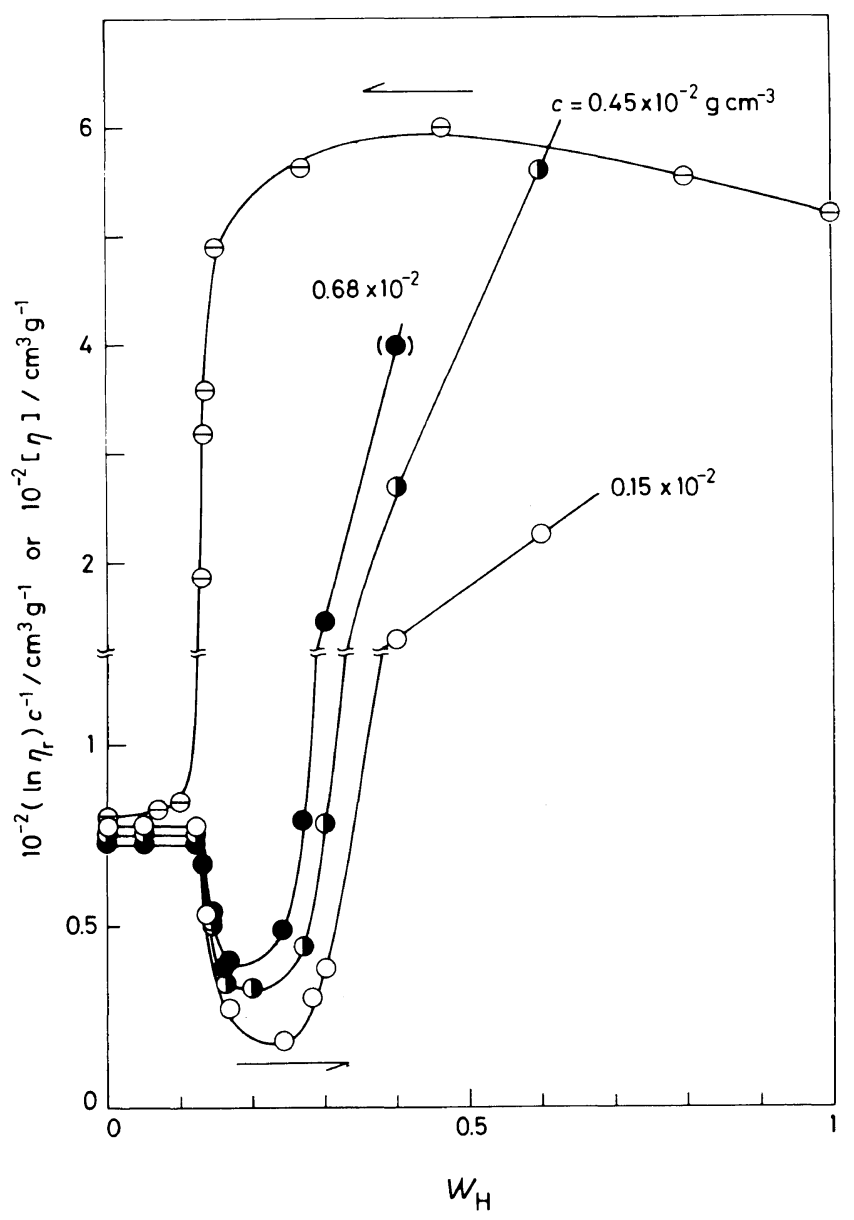

Figure 3. Composition dependence of $\left(\ln \eta_{\mathrm{r}}\right) / c(\Theta, O, \bigcirc)$ for sample S-197 in water-diluted solutions (lower arrow) at an elapsed time of $10 \mathrm{~h}$ and that of $[\eta](\ominus)$ for the same sample in DMSO-diluted solutions (upper arrow).

\section{Interpretation of Experimental Results}

(1) In Figure 3, the values of $\left(\ln \eta_{\mathrm{r}}\right) / c$ of waterdiluted solutions with $w_{\mathrm{H}}<0.13$ are independent of $w_{\mathbf{H}}$ and nearly so of $c$. This finding may be taken as indicating that neither interchain nor intrachain association takes place in these ranges of $w_{\mathrm{H}}$ and $c$. The time independence of $\left(\ln \eta_{\mathrm{r}}\right) / c$ in the solution with $w_{\mathrm{H}}=0.121$ (Figure 2) is consistent with this interpretation.

(2) In Figure 3, the breaks of the curves for water-diluted solutions at $w_{\mathrm{H}} \sim 0.13$ mark the onset of the above-mentioned association processes. The sharp decrease in $\left(\ln \eta_{\mathrm{r}}\right) / c$ following the breaks indicates the dominance of the chain collapse. The monotonic decline of the curves for $w_{\mathrm{H}}=0.140$ and
0.157 in Figure 2 confirms that this is true. However, the behavior of these curves should not be taken as totally due to chain collapsing. The leveling-off at their later stage is probably due to the counterbalance of intra and interchain associations.

(3) As $w_{\mathrm{H}}$ is increased, the interchain association of collapsed coils is enhanced and reaches a point at which the viscosity increase due to this effect is offset by the viscosity decrease due to chain collapsing. The minima of the curves for waterdiluted solutions in Figure 3 should correspond to such points.

(4) The data of Figure 3 allow a value of about $10 \mathrm{~cm}^{3} \mathrm{~g}^{-1}$ to be estimated for the limit of $\left(\ln \eta_{\mathrm{r}}\right) / c$ at infinite dilution in the vicinity of these minima. 


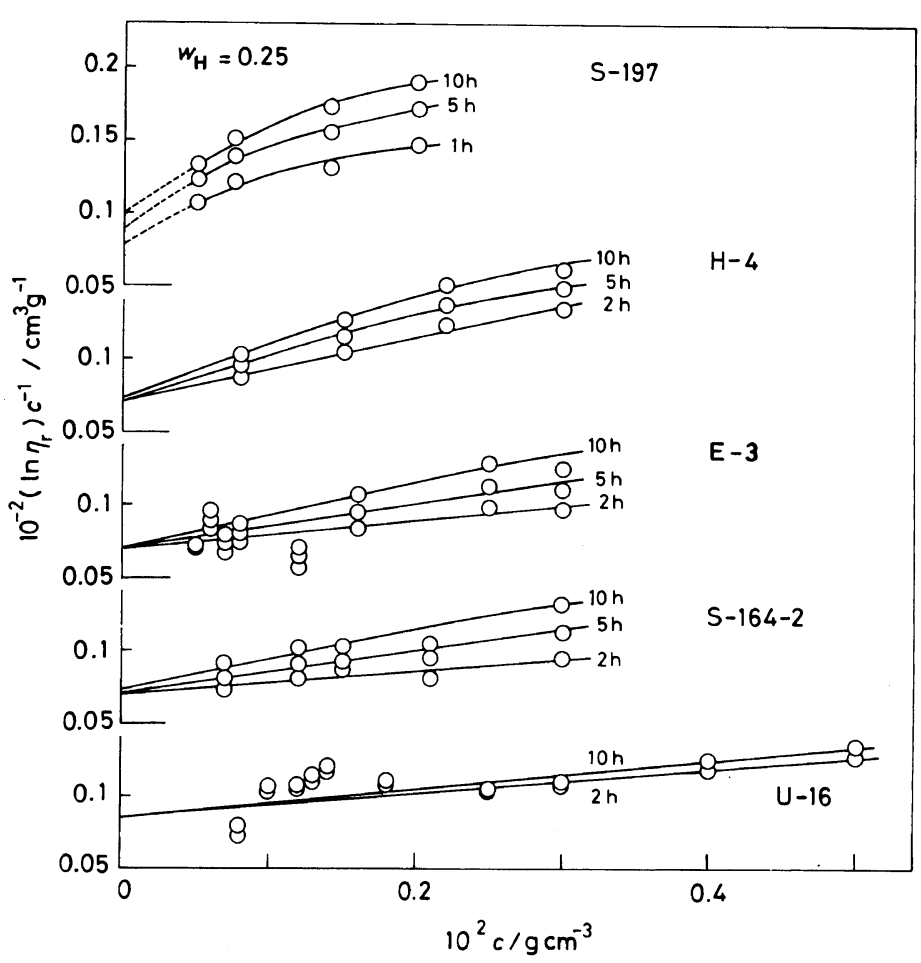

Figure 4. Plots of $\left(\ln \eta_{\mathrm{r}}\right) / c v s . c$ at fixed values of $t$ for five schizophyllan samples in the water+DMSO mixture of $w_{\mathbf{H}}=0.25$.

This limiting value is less than one seventh the intrinsic viscosity in DMSO, indicating how appreciably the schizophyllan random coil is shrunk when $w_{\mathrm{H}}$ is increased to 0.20 or more. Thus, it may be concluded that the marked upswing of the curves for water-diluted solutions in the region of $w_{\mathrm{H}}$ above 0.20 is almost entirely caused by enhanced interchain association accompanying an increase in $w_{\mathrm{H}}$. This conclusion is consistent with the behavior of the curves for $w_{\mathrm{H}}=0.240,0.270$, and 0.300 in Figure 2. It can be seen that, at such high $w_{\mathrm{H}}$, the formation of aggregates tend to surpass the chain collapse as the solution is allowed to stand longer.

(5) The data of Figure 1 shows a pronounced increase in $\left(\ln \eta_{\mathrm{r}}\right) / c$ with $c$, in contrast to the case of nonassociating polymers, in which $\left(\ln \eta_{\mathrm{r}}\right) / c$ usually decreases slightly with increasing $c$. From the curves in Figure 3 the infinite-dilution value of $\left(\ln \eta_{\mathrm{r}}\right) / c$ at $w_{\mathrm{H}}=0.168$ can be estimated to be not more than 20 $\mathrm{cm}^{3} \mathrm{~g}^{-1}$. The contribution of collapsed chains to $\left(\ln \eta_{\mathrm{r}}\right) / c$ at finite $c$ may remain at this order of magnitude, since, as noted above, the chain collapse may be a concentration-independent process. Thus, it may be concluded that the characteristic concentration dependence of $\left(\ln \eta_{\mathrm{r}}\right) / c$ displayed in Figure 1 arises almost entirely from interchain association. A point to note is that, even at this fairly low $w_{\mathrm{H}}$, there occurs a significant extent of interchain association.

\section{Determination of $[\eta]$}

The intrinsic viscosity $[\eta]$ of an isolated schizophyllan molecule in a water + DMSO mixture of known $w_{\mathrm{H}}$ at an elapsed time $t$ may be estimated if the $\left(\ln \eta_{\mathrm{r}}\right) / c v s$. time curve at that $w_{\mathrm{H}}$ is determined for a series of low $c$. The values of $[\eta]$ thus obtained for different $t$ afford information about the rate of chain collapse.

The $\left(\ln \eta_{\mathrm{r}}\right) / c v s . c$ plots for several fixed values of $t$ constructed from the data for five schizophyllan samples in the mixture of $w_{\mathbf{H}}=0.25$ are illustrated in Figure 4. It can be seen that, except for the highestmolecular weight sample S-197, the data points for a fixed $t$ fall approximately on a straight line and the 


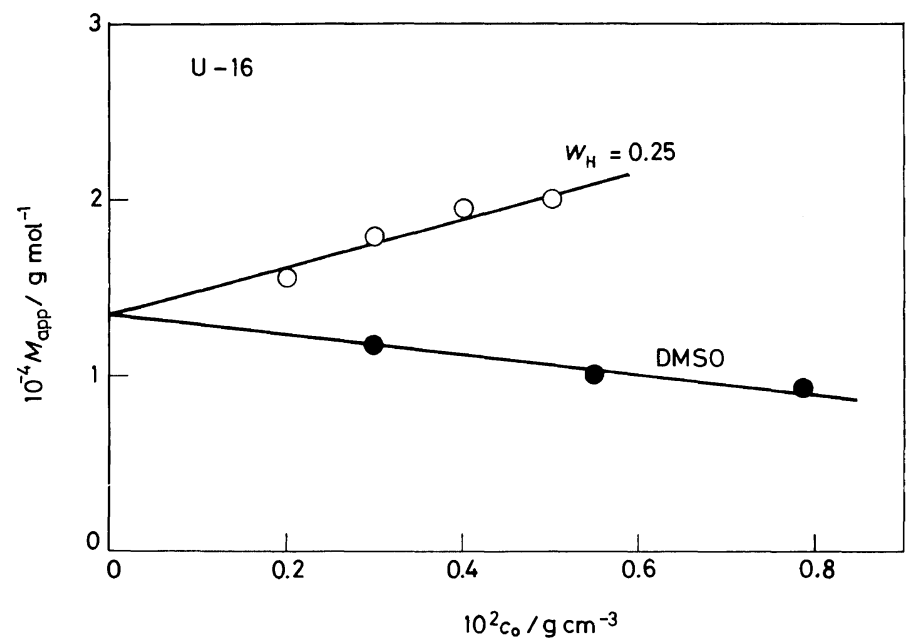

Figure 5. Plots of $M_{\text {app }} v s$. initial polymer concentration for sample U-16 in pure DMSO and the water+DMSO mixture of $w=0.25$.

lines for different $t \geqq 2 \mathrm{~h}$ lead to essentially the same $[\eta]$. This result indicates that, in the mixture of $w_{\mathrm{H}}=$ 0.25 , the collapsed chain at infinite dilution reaches an equilibrium size in about $2 \mathrm{~h}$ after a given DMSO solution has been diluted with water. The same was found to hold at $w_{\mathrm{H}}=0.20$. However, it must be noted that before coming down to a nearequilibrium value (about $10 \mathrm{~cm}^{3} \mathrm{~g}^{-1}$ for these $w_{\mathrm{H}}$ ) the intrinsic viscosity suffers an enormous decrease from the initial value in DMSO. This can be seen from the curves in Figures 1 and 2 showing almost discontinuous viscosity drops at the beginning of the process. Actually, these initial changes are combined effects of very fast inter and intrachain associations occurring soon after the preparation of water-diluted solutions.

The Huggins constant for the lines in Figure 4 is anomalously large. It is reasonable to attribute this feature substantially to interchain association. It should be noted that the formation of aggregates does occur even at very high dilutions, making it difficult to estimate $[\eta]$ as illustrated by the data for sample S-197 in Figure 4.

In Figure 5, the sedimentation equilibrium data for sample U-16 in pure DMSO and the mixture of $w_{\mathrm{H}}=0.25$ at $25^{\circ} \mathrm{C}$ are shown as $M_{\text {app }}$ plotted against initial polymer concentration $c_{0}$. The two plots give the same intercept, confirming molecular dispersion of sample U-16 at infinite dilution in this mixed solvent.

\section{Globular Conformation}

The values of $[\eta]$ for equilibrium collapsed chains taken from Figure $4^{*}$ and the corresponding data for the mixture of $w_{\mathrm{H}}=0.20$ are shown as a function of molecular weight (in DMSO) in Figure 6, where the molecular weight dependence of $[\eta]$ for schizophyllan in pure DMSO is shown for comparison. If allowance is made for the above-mentioned difficulty in the determination of $[\eta]$, the intrinsic viscosity in each of these mixtures may be regarded as independent of molecular weight, being about $11 \mathrm{~cm}^{3} \mathrm{~g}^{-1}$ for $w_{\mathrm{H}}=0.20$ and about $8 \mathrm{~cm}^{3} \mathrm{~g}^{-1}$ for $w_{\mathrm{H}}=0.25$. Thus, schizophyllan random coils in these mixtures are collapsed to globules with densities essentially independent of molecular weight.

The arrow in Figure 6 indicates $[\eta]$ calculated from Einstein's formula for a rigid sphere on the assumption that the hydrodynamic volume per gram of the globule is equal to the specific volume of schizophyllan. This value is about one fifth the experimental value for schizophyllan globules in the mixture of $w_{\mathrm{H}}=0.25$. Thus, these globules are not quite compact but $80 \%$ of their volumes are occupied by solvent molecules. Interestingly, a comparable occupied volume of solvent in a globule was reported a long time ago for a highly branched polysaccharide glycogen in $0.1 \mathrm{M}$ calcium chloride by Husemann, ${ }^{6}$ who showed that the values of $[\eta]$ of a series of glycogen samples ranging in molecular weight from $2 \times 10^{4}$ to $1.53 \times 10^{6}$ were about $8 \mathrm{~cm}^{3}$

* For sample S-197 the smallest of the intercepts was taken as the desired $[\eta]$. 


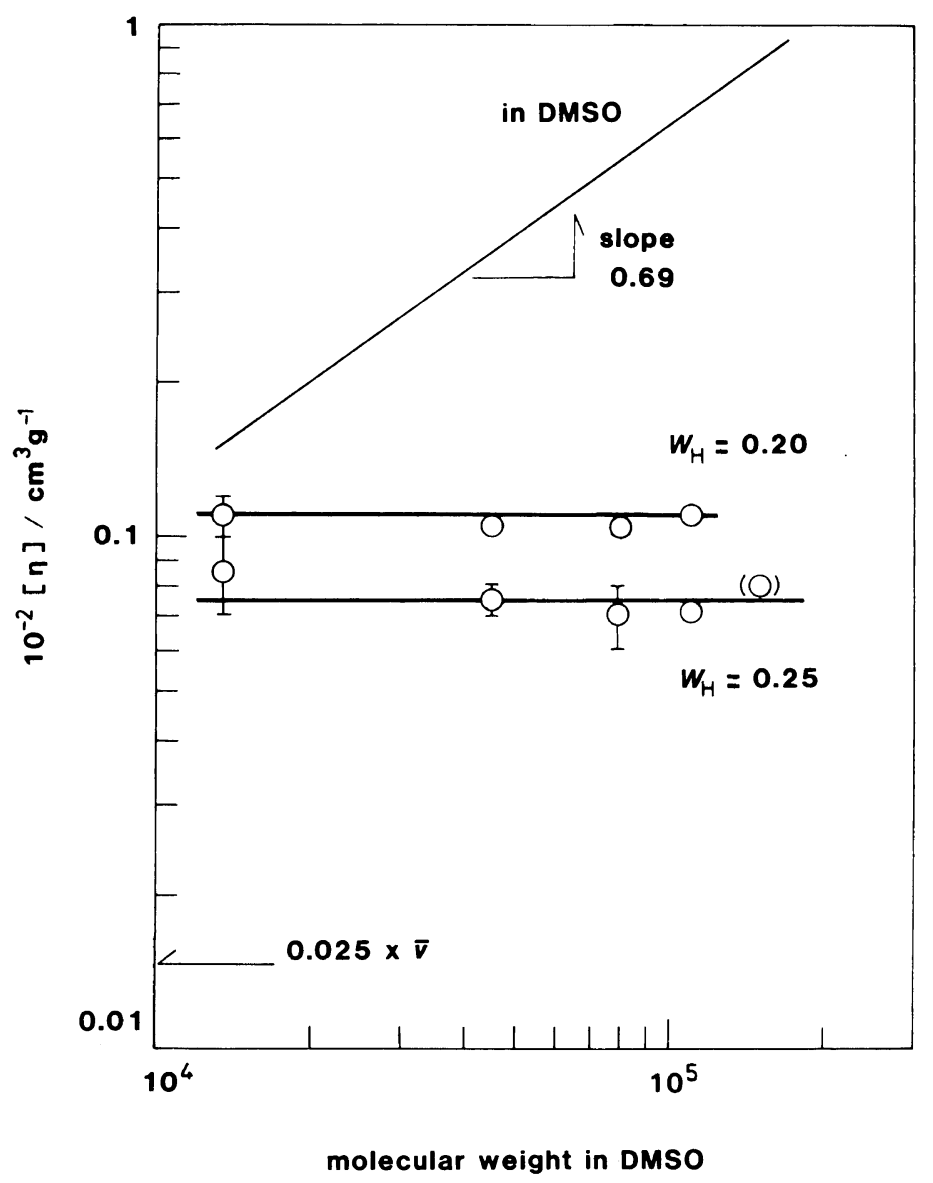

Figure 6. Molecular weight dependence of $[\eta]$ for equilibrium collapsed chains of schizophyllan in the water + DMSO mixtures of $w_{\mathrm{H}}=0.20$ and 0.25 . The solid line represents the $[\eta] v s$. molecular weight relation $^{3}$ for randomly coiled schizophyllan in DMSO, and the arrow indicates $[\eta]$ calculated from Einstein's formula for rigid spheres.

$\mathrm{g}^{-1}$.

Except for proteins, there are few demonstrations of globular polymer conformation, though not a few data $^{7-9}$ indicating chain collapses have been reported. Priel and Silberberg ${ }^{8}$ found that $[\eta]$ of poly(methacrylic acid) in mixtures of $0.002 \mathrm{~N}$ hydrochloric acid (a theta solvent for this polymer) and ethanol anomalously decreased when the mole fraction of ethanol was increased to 0.04. They found, however, that $[\eta]$ at this ethanol composition increased in proportion to the square root of molecular weight. This molecular weight dependence is not of the kind known for globules. Recently, Nishio et al. ${ }^{9}$ found that the statistical and hydrodynamic radii of a poly(acrylamide) sam- ple in mixtures of water and acetone very sharply decreased when the water composition of the binary solvent was lowered to about $60 \mathrm{vol} \%$, and concluded that these decreases in dimensions resulted from a coil-globule transition. However, this conclusion must be accepted with reservation, since these authors did not check the molecular weight dependence of the dimensions or $[\eta]$.

It does not seem relevant to discuss our finding of chain collapse on the basis of the current theory $y^{7,10-12}$ of coil-globule transition, since the formation of schizophyllan globules is due primarily to intramolecular hydrogen bonding of glucose residues, whereas the theory considers not such a specific interaction but attractive interactions of 


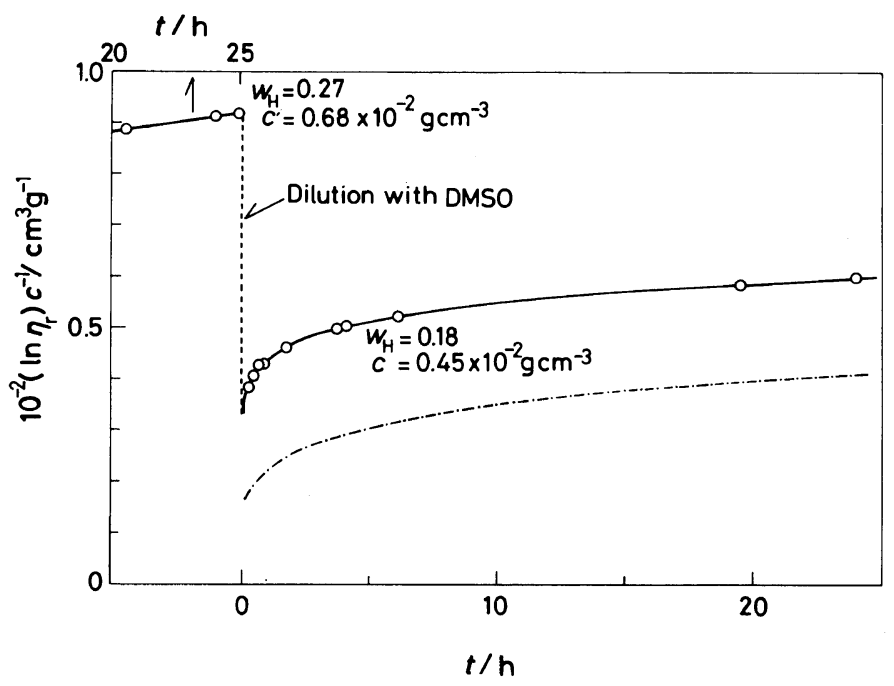

Figure 7. Time change in $\left(\ln \eta_{\mathrm{r}}\right) / c$ that occurred after a water-diluted solution of sample $\mathrm{S}-197\left(w_{\mathrm{H}}=0.27\right.$, $c=0.68 \times 10^{-2} \mathrm{~g} \mathrm{~cm}^{-3}$, and $t=25 \mathrm{~h}$ ) was diluted with DMSO to a solution with $w_{\mathrm{H}}=0.18$ and $c=$ $0.45 \times 10^{-2} \mathrm{~g} \mathrm{~cm}^{-3}$. The dot-dash line shows the change in $\left(\ln \eta_{\mathrm{r}}\right) / c$ of the water-diluted solution with the same $w_{\mathrm{H}}$ and $c$ as the rediluted solution.

the van der Waals type between chain segments to be the cause of chain collapsing. The schizophyllan globule may be better visualized as an intramolecularly crosslinked ${ }^{13}$ or highly branched ${ }^{6}$ polymer molecule.

\section{Remarks}

The solvent DMSO was added to some waterdiluted solutions to lower $w_{\mathbf{H}}$ after successive viscosity measurements were completed, and viscosity changes in the resulting solutions were determined as a function of time.

Figure 7 illustrates the data from such an experiment, in which the water-diluted solution with $w_{\mathrm{H}}=$ 0.27 and $c=0.68 \times 10^{-2} \mathrm{~g} \mathrm{~cm}^{-3}$ at an elapsed time of $25 \mathrm{~h}$ was diluted to a solution with $w_{\mathrm{H}}=0.18$ and $c=$ $0.45 \times 10^{-2} \mathrm{~g} \mathrm{~cm}^{-3}$. In this figure, $t$ for the "rediluted" solution is measured from the instant at which DMSO was added, and the dot-dash line shows the time change in $\left(\ln \eta_{\mathrm{r}}\right) / c$ of the waterdiluted solution with the same $w_{\mathbf{H}}$ and $c$ as the rediluted solution. It can be seen that a very fast decrease in $\left(\ln \eta_{\mathrm{r}}\right) / c$ at the beginning is followed by a gradual, monotonic increase. Interestingly, the latter process parallels the dot-dash line, but both do not appear to approach the same asymptotic level.

In another experiment in which redilution was made from $w_{\mathrm{H}}=0.27$ and $c=0.45 \times 10^{-2} \mathrm{~g} \mathrm{~cm}^{-3}$ to
$w_{\mathrm{H}}=0.26$ and $c=0.15 \times 10^{-2} \mathrm{~g} \mathrm{~cm}^{-3},\left(\ln \eta_{\mathrm{r}}\right) / c$ decreased gradually with $t$ and, at $t=20 \mathrm{~h}$, leveled off at a value much higher than that for the waterdiluted solution at $t=20 \mathrm{~h}$ having the same $w_{\mathrm{H}}$ and $c$ as the rediluted solution.

Further, when two water-diluted solutions with $w_{\mathrm{H}}$ far above 0.13 were rediluted to $w_{\mathrm{H}}=0.07$, $\left(\ln \eta_{\mathrm{r}}\right) / c$ very rapidly resumed the values for waterdiluted solutions in the region of $w_{\mathrm{H}}$ below 0.13 .

These experimental data, though fragmentary, indicate that the viscosity of water-diluted solutions is irreversible or reversible with respect to the change in $w_{\mathrm{H}}$, depending on whether the redilution is made to $w_{\mathrm{H}}$ above or below 0.13 . Note that this $w_{\mathrm{H}}$ is the critical composition of the solvent for the onset of both inter and intrachain associations. Discussion on the complex viscosity changes accompanying redilution will be deferred until more experimental data are accumulated.

Acknowledgment. We acknowledge with thanks a grant from Taito Co.

\section{REFERENCES}

1. T. Norisuye, T. Yanaki, and H. Fujita, J. Polym. Sci., Polym. Phys. Ed., 18, 547 (1980).

2. T. Yanaki, T. Norisuye, and H. Fujita, Macromolecules, 13, 1462 (1980). 
3. Y. Kashiwagi, T. Norisuye, and H. Fujita, Macromolecules, 14, 1220 (1981).

4. T. Sato, T. Norisuye, and H. Fujita, Macromolecules, in press.

5. T. Sato, T. Norisuye, and H. Fujita, Carbohydr. Res., 95, 195 (1981).

6. E. Husemann, J. Prakt. Chem. N. F., 158, 163 (1941).

7. C. Williams, F. Brochard, and H. Frish, Ann. Rev. Phys. Chem., 32, 433 (1981).

8. Z. Priel and A. Silberberg, J. Polym. Sci., A-2, 8, 713
(1970).

9. I. Nishio, S. T. Sun, G. Swislow, and T. Tanaka, Nature, 281, 208 (1979).

10. P. G. de Gennes, J. Phys. Lett. (Paris), 36, L55 (1975); 39, L299 (1978).

11. M. Daoud and G. Jannink, J. Phys. (Paris), 37, 973 (1976).

12. I. C. Sanchez, Macromolecules, 12, 980 (1979).

13. G. Allen, J. Burgess, S. F. Edwards, and D. J. Walsh, Proc. R. Soc. London, Ser. A, 334, 477 (1973). 